\title{
Effects of behavioural activation on the neural basis of other perspective self-referential processing in subthreshold depression: a functional magnetic resonance imaging study
}

\author{
S. Shiota ${ }^{1}$, Y. Okamoto ${ }^{1 *}$, G. Okada ${ }^{1}$, K. Takagaki ${ }^{1}$, M. Takamura ${ }^{1}$, A. Mori ${ }^{1}$, S. Yokoyama ${ }^{1}$, \\ Y. Nishiyama ${ }^{1}$, R. Jinnin ${ }^{1}$, R. I. Hashimoto ${ }^{2,3}$ and S. Yamawaki ${ }^{1}$ \\ ${ }^{1}$ Department of Psychiatry and Neurosciences, Graduate School of Biomedical and Health Sciences, Hiroshima University, Hiroshima, Japan \\ ${ }^{2}$ Department of Language Sciences, Graduate School of Humanities, Tokyo Metropolitan University, Tokyo, Japan \\ ${ }^{3}$ Medical Institute of Developmental Disabilities Research, Showa University, Tokyo, Japan
}

Background. It has been demonstrated that negatively distorted self-referential processing, in which individuals evaluate one's own self, is a pathogenic mechanism in subthreshold depression that has a considerable impact on the quality of life and carries an elevated risk of developing major depression. Behavioural activation (BA) is an effective intervention for depression, including subthreshold depression. However, brain mechanisms underlying BA are not fully understood. We sought to examine the effect of BA on neural activation during other perspective self-referential processing in subthreshold depression.

Method. A total of 56 subjects underwent functional magnetic resonance imaging scans during a self-referential task with two viewpoints (self/other) and two emotional valences (positive/negative) on two occasions. Between scans, while the intervention group $(n=27)$ received BA therapy, the control group $(n=29)$ did not.

Results. The intervention group showed improvement in depressive symptoms, increased activation in the dorsal medial prefrontal cortex (dmPFC), and increased reaction times during other perspective self-referential processing for positive words after the intervention. Also, there was a positive correlation between increased activation in the dmPFC and improvement of depressive symptoms. Additionally, there was a positive correlation between improvement of depressive symptoms and increased reaction times.

Conclusions. BA increased dmPFC activation during other perspective self-referential processing with improvement of depressive symptoms and increased reaction times which were associated with improvement of self-monitoring function. Our results suggest that BA improved depressive symptoms and objective monitoring function for subthreshold depression.

Received 22 March 2016; Revised 12 October 2016; Accepted 14 October 2016; First published online 29 November 2016

Key words: Behavioural activation, dorsal medial prefrontal cortex, monitoring function, subthreshold depression.

\section{Introduction}

Subthreshold depression is defined as clinically significant depressive symptoms which do not fulfill diagnostic criteria for a major depressive episode (Bertha \& Balazs, 2013). Subthreshold depression can lead to serious functional impairment, including a negative impact on academic performance and social activity, particularly in adolescence (Bertha \& Balazs, 2013). Subthreshold depression is associated with an elevated

* Address for correspondence: Y. Okamoto, M.D., Ph.D., Department of Psychiatry and Neurosciences, Graduate School of Biomedical and Health Sciences, Hiroshima University, Hiroshima, Japan.

(Email: oy@hiroshima-u.ac.jp) risk of developing a major depressive episode (Bertha \& Balazs, 2013).

Given these concerns, it is very important to elucidate the pathogenic mechanisms underlying subthreshold depression and to develop effective interventions. Although pharmacotherapy is widely provided, use of selective serotonin reuptake inhibitors in youth can have aversive effects, such as increased suicidality (Hammad et al. 2006). Consequently, psychotherapy is preferred for treatment of youth depression. Cognitive-behavioural therapy (CBT) has demonstrated efficacy with this population (Cuijpers et al. 2007).

In particular, behavioural activation (BA) is one key component of CBT for depression, and is an effective intervention for the treatment of major depression

This is an Open Access article, distributed under the terms of the Creative Commons Attribution licence (http://creativecommons.org/licenses/by/4.0/), which permits unrestricted re-use, distribution, and reproduction in any medium, provided the original work is properly cited. 
(Jacobson et al. 1996). Based on the approach of Lewinsohn \& Grosscup (1980), BA is focused on enhancement of self-monitoring, increasing healthy goal-oriented behaviour, and increasing environmental reward frequency. In the course of BA interventions, participants monitor and assess their daily activities and work to change their habitual behaviours, such that pleasant events are increased and depressive symptoms are improved (Jacobson et al. 1996). Recently, we carried out a randomized controlled trial of BA for subthreshold depression in college students, demonstrating that BA is an effective intervention for people with subthreshold depression (Takagaki et al. 2016). However, brain mechanisms underlying BA intervention are not fully understood.

A few studies have investigated BA-related brain mechanisms in depression using tasks that probe emotion regulation (Dichter et al. 2010) and reward processing (Dichter et al. 2009). Our previous study demonstrated that BA for subthreshold depression leads to functional changes in the left ventrolateral prefrontal cortex (PFC) and angular gyrus during loss anticipation in a monetary incentive task (Mori et al. 2016).

Material related to the self is more readily remembered than material related to others, which is referred to as self-referential processing (Rogers et al. 1977; Symons et al. 1997). Especially, self-referential processing with other perspective is related to a person's objective monitoring function (Davis et al. 1996; Galinsky \& Ku, 2004). It has been demonstrated that negatively distorted self-perspective self-reference, in which individuals evaluate one's own self using selfviewpoint, is a pathogenic mechanism in both major depression and subthreshold depression (Kuiper \& Derry, 1982), and it has been suggested that there are abnormalities in brain activation that accompany such negatively distorted self-perspective (Calni et al. 2004; Yoshimura et al. 2010). In addition, individuals with depression also show impairments in positive other perspective self-reference, where individuals evaluate one's own self using his or her friend's viewpoint (Surguladze et al. 2004), and such altered selfreferential processing leads to the maintenance of depressive symptoms (Weightman et al. 2014). Our previous study demonstrated that symptom improvement in CBT was associated with activation changes in the medial PFC (mPFC) during negative selfreference from the self-perspective in depression (Yoshimura et al. 2014).

The original purpose of BA was to increase access to positively reinforcing activities, increasing the availability of rewards and modifying behaviours to elicit changes in depressive symptoms. However, according to a previous study (Jacobson et al. 1996), BA also significantly improved the score in the Automatic Thoughts Questionnaire (ATQ) related to the selfconcept. The ATQ assesses a person's negative automatic thoughts related to negative self-concepts (Hollon \& Kendall, 1980). Based on these findings, we speculated that BA might modify the self-concept. A notable point of the current study was that participants repeatedly evaluated their behaviours and their results during the behavioural experiments. Lewinsohn \& Grosscup (1980) have suggested that participants enhance their monitoring function as a consequence of repeated evaluation of their own behaviours and results during BA. Self-referential processing with an other perspective is related to a person's objective monitoring function (Davis et al. 1996; Galinsky \& Ku, 2004). Therefore, we hypothesize that BA enhances other perspective self-referential processing, which is a part of the self-concept that is associated with changes in mPFC activation. However, there are no published studies that have specifically focused on brain function changes related to other perspective self-reference, which is also known to be associated with mPFC activation (Ruby \& Decety, 2001) in depression and subthreshold depression during the course of treatment. In the present study, we carried out a BA intervention for university students with subthreshold depression in order to examine whether BA might modify mPFC activity during referential processing. We employed the modified self-reference functional magnetic resonance imaging (fMRI) task adopted by Hashimoto et al. (2016) which was designed to examine effects of perspective on brain activation for self- and other referential processing. We hypothesized that successful BA intervention may (1) decrease negative other perspective selfreference, corresponding to $\mathrm{mPFC}$ activation and (2) increase positive other perspective self-reference, also corresponding to $\mathrm{MPFC}$ activation.

\section{Method \\ Participants}

Participants were recruited over a 2-year period between 2013 and 2014 from Hiroshima University, by screening with the Japanese version of the Beck Depression Inventory-II (BDI-II) (Kojima \& Furukawa, 2003) and the Japanese version of the structured clinical interview (Composite International Diagnostic Interview; Kawakami et al. 2005). The inclusion criterion was BDI-II scores greater than or equal to 10 . The exclusion criteria were major depressive episode within the past year, lifetime history of bipolar disorder, presently undergoing psychopharmacological or psychological treatment, and the possibility of an acute suicide 
attempt. A total of 61 students (mean age $=18.2$ years, S.D. $=0.4$ years, 40 males, 21 females) with subthreshold depression were enrolled in the randomized controlled trial of BA in the second year. Individuals were then randomly allocated to an intervention group $(n=30$, mean age $=18.2$ years, s.D. $=0.4$ years, 19 males, 11 females) or to a no-treatment control group $(n=31$, mean age $=18.2$ years, s.D. $=0.4$ years, 21 males, 10 females). One participant in the intervention group and another in the no-treatment control group did not participate in the fMRI study. In all, 29 participants in the intervention group (mean age $=18.2$ years, S.D. $=0.4$ years, 19 males, 10 females) and 30 participants in the no-treatment control group (mean age $=18.2$ years, S.D. $=0.4$ years, 21 males, nine females) participated in the fMRI study. Three participants were excluded from fMRI analyses, because two of them took medication and the other did not yield usable data. All told, fMRI and behavioural data of 27 participants in the intervention group and 29 individuals in the control group were analysed. The ethics committee of Hiroshima University approved the study protocol. Prior to the study, written informed consent was obtained from all participants.

\section{Experimental design}

Freshmen attending Hiroshima University were recruited for the randomized controlled trial (Takagaki et al. 2016). A total of 59 subjects underwent fMRI scans during a referential task with two viewpoints (self/other) and two emotional valences (positive/negative) (Hashimoto et al. 2016). For the intervention group, the scan occurred both before and after BA intervention, and the control group was also scanned twice, with an interval of 5 weeks without any intervention.

\section{Treatment protocol}

Individuals in the intervention group participated in the BA programme (Takagaki et al. 2016). The BA intervention is described in detail elsewhere (Takagaki et al. 2016). Briefly, the BA intervention included five weekly sessions, each of $60 \mathrm{~min}$ duration. In the 1st session, participants received psycho-education about depression and BA and set long-term and short-term goals. The concept of activity monitoring was introduced. In the 2nd session, participants developed a hierarchy of about 10 tasks, were introduced to the concept of behavioural experiments, and received instruction around how to increase scheduled activities. The 3rd and 4th sessions continued to focus on behavioural experiments and increasing scheduled activities. In the 5th session, participants assessed improvements in activity-monitoring ability compared with the 1st session, reviewed progress to date, and built a plan for self-management in stressful situations. This treatment protocol involved homework for all sessions. Participants monitored their behaviours during all 5 weeks of the programme.

\section{Evaluation}

Effects of BA on other perspective self-referential processing were measured using an fMRI task. Behavioural data included reaction times and judgment ratios. Reaction time was the average duration of participants' responses to stimuli and judgment ratio was the average number of 'yes' response counts during each emotional valence (positive/negative) condition, as described below. Depression was assessed using the BDI-II at pre- and post-treatment. Additionally, we calculated $\Delta$ brain activation (posttreatment minus pre-treatment) and percentage change on BDI-II [(pre-treatment minus post-treatment) divided by pre-treatment], as well as percentage change on reaction times [(post-treatment minus pretreatment) divided by pre-treatment] and percentage change on judgment ratios [(post-treatment minus pretreatment) divided by pre-treatment]. We used percentage change scores in the present study because of their merits for correlation analyses. Reaction times, brain activation and BDI-II scores had different value ranges and the percentage change score can adjust for differences in the range of value of different variables (Tan \& Michel, 2011). Providing information related to the content and precision of scores helps the meaningful interpretation of raw scores (Tong \& Kolen, 2010). Analysis of data from clinical trials often uses percentage change scores and raw change scores to adjust treatment responses to the baseline (Kaiser, 1989). Additionally, the percentage change score can be adapted for ratio scales (Russell, 2000). If the variable were a ratio scale, then pre-scores and changed scores would be correlated (Sugimoto, 2008). We performed correlation analyses that included prescores and changed scores for different variables. Furthermore, we assessed normal distribution using skewness and kurtosis (Kim, 2013). It is known that distribution of small samples $(n<50)$ would be nonnormal if the absolute $z$-scores for either skewness or kurtosis were larger than 1.96 (Kim, 2013). To examine intervention effects, we performed correlation analyses that included percentage change on BDI-II and $\Delta$ brain activation, percentage change on reaction times, and percentage change on judgment ratios, respectively.

\section{fMRI task}

The fMRI task (Hashimoto et al. 2016) included five judgment conditions: other perspective self-judgment 
condition (OS); other perspective other judgment condition (OO); self-perspective self-judgment condition (SS); self-perspective other judgment condition (SO); and control judgment (word recognition) condition (Cont). Before the first scanning, participants were asked to name three of their friends of the same gender. Next, participants ranked these friends according to closeness. The second closest friend was used as the 'other' during the experiment. In OS condition trials, participants judged the presented positive (OS-P) or negative (OS-N) trait words using his or her friend's viewpoint, as applicable to one's own self [e.g. 'Does Bob think you are calm?' (OS-P)]. In OO condition trials, participants judged the presented positive (OO-P) or negative (OO-N) trait words using his or her friend's viewpoint, as applicable to his or her friend [e.g. 'Does Bob think Bob is calm?' (OO-P)]. In SS condition trials, participants judged positive (SS-P) or negative (SS-N) trait words using the self-viewpoint, applicable to one's own self [e.g. 'Do you think you are calm?' (SS-P)]. In SO condition trials, participants judged positive (SO-P) or negative (SO-N) trait words using the self-viewpoint, as applicable to his or her friend [e.g. 'Do you think Bob is calm?' (SO-P)]. Finally, in control (Cont) condition trials, participants judged positive (Cont-P) or negative (Cont-N) words as to whether or not they could understand their meanings. For all conditions, participants made a 'yes' or 'no' response by pressing a button with their right index or middle fingers respectively. Button presses were recorded using an MRIcompatible keypad (4 Side Button Cylinder; Current Designs, USA). The average number of 'yes' responses in each condition was defined as the judgment ratio (Yoshimura et al. 2014) and reaction time was defined as the average latency of participant's responses after adjective words were displayed on the screen (Sheppard \& Teasdale, 2000). Participants performed each condition eight times, and each condition included four blocks. At the onset of each block, a fixation cross was displayed for $1000 \mathrm{~ms}$, followed by an instruction cue presented for $3000 \mathrm{~ms}$ (e.g. 'selfviewpoint self-judgment'). Each block consisted of five trials, each consisting of a fixation cross displayed for $1000 \mathrm{~ms}$ followed by an adjective displayed for $3000 \mathrm{~ms}$ and the participant's response. A fixation point as an intermission between blocks was displayed for $4000 \mathrm{~ms}$, and then the instruction cue for the next block was presented. The duration of each block was $28 \mathrm{~s}$. To control for order effects, blocks within a run were presented in a pseudo-random order, with no two consecutive blocks featuring the same instructions. The total time for the task was $1120 \mathrm{~s}$. Both judgment ratio and reaction time were recorded using
Presentation software (Neurobehavioral Systems, USA).

\section{Stimuli}

Word stimuli were selected from Anderson's list of personality-trait words (Anderson, 1968) and Bochner and Van-zyl's list of personality-trait words (Bochner \& Van-zyl, 1985). The words in both lists were translated into Japanese (Yoshimura et al. 2010, 2014; Hashimoto et al. 2016). The top 200 words were selected for frequency using rank of Google searches as a criterion. Each adjective word was presented once randomly during the fMRI task.

\section{MRI acquisition}

MRI scanning was performed using a Verio 3.0T device (Siemens AG, Germany). A time-course series of 536 scans were acquired with T2*-weighted, gradient echo, echo-planar imaging sequences. Each volume consisted of 38 slices, with a slice thickness of $3.8 \mathrm{~mm}$ with no gap. The repetition time (TR) was $2000 \mathrm{~ms}$, the echo time (TE) was $25 \mathrm{~ms}$ and flip angle was $80^{\circ}$. The field of view (FOV) was 240 $\mathrm{mm}$, and the voxel size was $3.8 \times 3.8 \times 3.8 \mathrm{~mm}$. After functional scanning, structural scans were acquired using a T1-weight gradient echo pulse sequence $\left(\mathrm{TR}=2300 \mathrm{~ms}, \mathrm{TE}=2.98 \mathrm{~ms}\right.$, flip angle $=9^{\circ}$, FOV $=$ $256 \mathrm{~mm}$, voxel size $=1 \times 1 \times 1 \mathrm{~mm}$ ), which facilitated localization.

\section{Behavioural data analysis}

We conducted a three-way mixed ANOVA using group (intervention group $v$. control group) as a between-subjects factor, and time (pre-treatment $v$. post-treatment) and emotional valence (positive $v$. negative) as within-subjects factors, for judgment ratio and reaction time in the OS condition.

\section{fMRI data analysis}

Image processing and statistical analysis were carried out using Statistical Parametric Mapping (SPM8) software (Wellcome Department of Cognitive Neurology, UK). The first five volumes of the fMRI run were discarded to allow for T1 stabilization. All of the remaining volumes were slice timing corrected, realigned to the mean volume to correct for head motion, spatially normalized using the Montreal Neurological Institute (MNI) T1 template, and smoothed with 8-mm fullwidth, half-maximum Gaussian filter. A whole-brain voxel-by-voxel multiple linear regression model was employed at the individual participant level. Each condition was modelled using a box-car function convolved with a canonical haemodynamic response 
function. The realignment parameters were also included in the models as covariates of no interest. To evaluate brain activation related to OS, OO, SS and $\mathrm{SO}$ conditions for both positive and negative valence, we created eight contrasts ('OS-P minus Cont-P', 'OS-N minus Cont-N', 'OO-P minus Cont-P', 'OO-N minus Cont-N', 'SS-P minus Cont-P', 'SS-N minus Cont- $\mathrm{N}$ ', 'SO-P minus Cont- $\mathrm{P}^{\prime}$ and 'SO-N minus Cont- $\mathrm{N}^{\prime}$ ) in the first-level analysis for each participant. These contrasts were submitted to group analysis using a random-effect model. First, onesample $t$ tests were performed for all participants to assess the overall effect of each contrast, using the contrasts from pre-treatment experimental sessions. Second, two-sample $t$ tests (intervention group $v$. control group) were performed by using subtraction images of contrast in the OS condition (post-minus pre-, i.e. $\Delta$ brain activation) to assess the effect of BA on brain activity. Based on our hypothesis, we used the $\mathrm{mPFC}$ as the a priori region of interest (ROI) based on previous work (Ochsner et al. 2005; Yoshimura et al. 2010, 2014). According to our hypothesis, mPFC activation was expected to change in association with changes in other perspective selfreferential processing. We conducted ROI analyses using the data of a previous functional brain imaging study of other perspective self-referential processing, in order to validate our hypothesis. The ROI was defined on the basis of a functional brain imaging study by Ruby \& Decety (2001) that demonstrated the involvement of ROI in other perspective self-referential tasks (6 mm radius sphere, centre at MNI coordinates $x$ $=4, y=50, z=40$ ). Brain activations were reported if they exceeded $p<0.001$ (uncorrected) at the single voxel level, and $p<0.05$ [family-wise error (FWE) small volume corrected within the ROI] at the cluster level.

\section{Results}

\section{Gender difference}

There were significant differences in the number of males and females $\left(\chi^{2}=7.45, p<0.05\right)$.

\section{Normality test}

We conducted correlation analyses that included preand changed scores of each variable, in order to confirm if these variables were a ratio scale. In the intervention group, there were significant correlations between pre-BDI-II scores and changes in BDI-II scores $(r=0.63, p<0.0001)$, and between pre-reaction time scores and changes in reaction time scores $(r=-0.50$, $p<0.001)$. In the control group, there was a significant correlation between pre-BDI-II scores and changes in BDI-II scores $(r=0.48, p<0.001)$, and a significant correlation between pre-reaction time scores and changes in reaction time scores $(r=-0.44, p<0.005)$. Second, we conducted a normality test using skewness and kurtosis (Kim, 2013). For the intervention group percentage change in BDI-II: skewness $=0.89<1.96$, kurtosis $=0.008<1.96$; percentage change in reaction time: skewness $=0.20<1.96$, kurtosis $=0.59<1.96$. For the control group - percentage change in the BDI-II: skewness $=3.18>1.96$, kurtosis $=12.51>1.96$; percentage change in reaction time: skewness $=0.54<1.96$, kurtosis $=0.75<1.96$. Results indicated that only the percentage change in BDI-II of the control group was not normally distributed.

\section{Psychological and behavioural data}

Table 1 shows psychological and behavioural data. The group $\times$ time interaction was significant for the BDI-II $\left(F_{1,54}=10.359, p<0.01, \eta^{2}=0.161\right)$. Depressive symptoms were significantly improved in the BA intervention group compared with the control group. Concerning reaction times, the three-way interaction between group, time and valence was significant only in the OS condition $\left(F_{1,54}=7.896, p<0.01, \eta^{2}=\right.$ 0.128). Post-hoc analysis revealed that there was a significant group difference at pre-session in OS-N $\left(p<0.05, \eta^{2}=0.074\right)$, but not in OS-P. In contrast, there was a significant group difference at post-session in OS-P difference $\left(p<0.05, \eta^{2}=0.073\right)$, but not in OS-N. Therefore, the intervention group demonstrated longer reaction times at post-treatment in OS-P. There were no significant main effects or interactions in judgment ratios between group, time or emotional valence in the OS condition.

\section{fMRI data}

\section{Overall effect of each contrast}

Table 2 shows the results of whole-brain one-sample $t$ tests conducted for all subthreshold depressive participants, for each contrast. In OS-P, the left superior $\mathrm{mPFC}$ (Brodmann area A10), left mPFC (Brodmann area 8), left angular gyrus (Brodmann area 39), right cerebellum and left precuneus (Brodmann area 31) showed significant activation. In OS-N, the left superior mPFC (Brodmann area 8), left mPFC (Brodmann area 8), left posterior cingulum (Brodmann area 23), left angular gyrus (Brodmann area 39) and right cerebellum showed significant activation. Significant mPFC activation was shown in all conditions for both positive and negative words.

\section{Effect of $B A$}

In accordance with our a priori hypothesis, we restricted these analyses to the $\mathrm{mPFC}$. The intervention group showed a significant increase in left 
Table 1. Scores of BDI-II, judgment ratio and reaction time during four judgment conditions on two emotional valences at pre-/post-BA intervention

\begin{tabular}{|c|c|c|c|c|}
\hline & \multicolumn{2}{|c|}{ Intervention group $(n=27)$} & \multicolumn{2}{|c|}{ Control group $(n=29)$} \\
\hline & Pre (baseline) & Post (following BA) & Pre (baseline) & Post (following BA) \\
\hline BDI-II & $12.7(5.6)$ & $7.3(6.3)$ & $13.7(5.0)$ & $12.8(6.8)$ \\
\hline \multicolumn{5}{|l|}{ Judgment ratio } \\
\hline Other perspective self-judgment/positive (OS-P) & $8.1(3.6)$ & $7.8(3.7)$ & $8.9(2.8)$ & $8.5(3.4)$ \\
\hline Other perspective self-judgment/negative (OS-N) & $4.9(3.7)$ & $5.1(4.0)$ & $6.2(3.6)$ & $6.5(4.4)$ \\
\hline Other perspective other judgment/positive (OO-P) & $9.6(3.4)$ & $9.5(3.5)$ & $11.0(3.9)$ & $11.3(4.0)$ \\
\hline Other perspective other judgment/negative (OO-N) & $3.3(3.7)$ & $3.4(2.9)$ & $4.6(3.2)$ & $4.7(2.7)$ \\
\hline Self-perspective self-judgment/positive (SS-P) & $6.0(3.7)$ & $6.7(4.0)$ & $8.0(3.6)$ & $7.8(3.7)$ \\
\hline Self-perspective self-judgment/negative (SS-N) & $10.0(3.9)$ & $10.3(4.6)$ & $9.6(3.5)$ & $10.5(4.0)$ \\
\hline Self-perspective other judgment/positive (SO-P) & $12.2(3.6)$ & $12.7(4.0)$ & $13.1(3.5)$ & $12.3(3.4)$ \\
\hline Self-perspective other judgment/negative (SO-N) & $2.7(2.8)$ & $2.3(1.8)$ & $3.7(3.8)$ & $4.1(2.8)$ \\
\hline \multicolumn{5}{|l|}{ Reaction time, $\mathrm{ms}$} \\
\hline Other perspective self-judgment/positive (OS-P) & $1624.9(354.4)$ & $1706.4(331.4)$ & $1579.5(211.6)$ & $1546.5(226.1)$ \\
\hline Other perspective self-judgment/negative (OS-N) & $1668.1(330.4)$ & $1669.9(352.1)$ & $1514.3(209.4)$ & $1614.3(278.7)$ \\
\hline Other perspective other judgment/positive (OO-P) & $1739.0(347.4)$ & $1734.0(349.4)$ & $1594.0(263.2)$ & $1582.0(238.3)$ \\
\hline Other perspective other judgment/negative (OO-N) & $1632.0(389.3)$ & $1560.0(333.4)$ & $1577.0(269.4)$ & $1526.0(239.4)$ \\
\hline Self-perspective self-judgment/positive (SS-P) & $1558.3(246.6)$ & $1548.3(307.6)$ & $1512.1(198.4)$ & $1471.9(260.6)$ \\
\hline Self-perspective self-judgment/negative (SS-N) & $1664.3(325.0)$ & $1631.7(283.4)$ & $1525.9(205.8)$ & $1568.8(264.9)$ \\
\hline Self-perspective other judgment/positive (SO-P) & $1615.0(352.4)$ & $1632.0(291.7)$ & $1524.0(246.5)$ & $1590.0(276.7)$ \\
\hline Self-perspective other judgment/negative (SO-N) & $1631.0(340.1)$ & $1581.0(281.1)$ & $1469.0(245.8)$ & $1529.0(261.4)$ \\
\hline
\end{tabular}

Data are given as mean (standard deviation).

BDI-II, Beck Depression Inventory-II; BA, behavioural activation.

dorsal (superior) mPFC activation in the OS-P $(x=-2$, $y=48, z=50, t=3.24$, cluster size $=70$, cluster level FWE-small volume corrected $p=0.016$ ) compared with the control group (see Fig. 1). There was no significant difference in OS-N activation in the mPFC between the intervention and control groups.

\section{Correlation analysis}

We extracted the eigenvariate value of the dorsal medial PFC (dmPFC) during OS-P in both the intervention and control groups. The eigenvariate value of $\mathrm{dmPFC}$ in OS-P was extracted from subtraction images of contrast using ROI. We conducted a correlation analysis to examine the relationship between $\Delta$ brain activation in the dmPFC during OS-P and percentage change on the BDI-II. We found a statistically significant positive correlation between $\triangle$ brain activation in the dmPFC and percentage change on the BDI-II in the intervention group ( $r=0.45, p<0.05$, Fig. $2 a)$, but not in the control group $(r=0.12, p=0.53$, N.s.). In addition, we conducted a correlation analysis between percentage change on reaction times and percentage change on BDI-II. We found a statistically significant positive correlation between these indicators in the intervention group ( $r=0.51, p<0.01$, Fig. $2 b)$, but not the control group ( $r=0.15, p=0.45$, N.s.).

\section{Multiple regression}

BA increased $\mathrm{dmPFC}$ activation and reaction time during OS-P, which was associated with improvements in depressive symptoms. Regions of the mPFC might be related to aspects of cognitive control and scale with reaction time on task (Grinband et al. 2008). Therefore, we performed an additional multiple regression analysis during OS-P with $\Delta$ brain activation as the dependent variable and percentage change score in reaction times as independent variables, in order to identify activation in cortical regions that were associated with reaction time. As in other analyses, we set the statistical threshold at $p<0.001$ (uncorrected) at the single voxel level, and $p<0.05$ (FWE-small volume corrected within the mPFC ROI) at the cluster level. However, there were no areas showing significant positive or negative correlations between reaction time and brain activation during OS-P.

\section{Functional connectivity change correlated with intervention effect}

We performed a generalized pycho-physiological interaction analysis (gPPI; http://www.nitrc.org/projects/gppi: Mclaren et al. 2012) with $\mathrm{mPFC}$ as the seed 
Table 2. Brain regions exhibiting significant activation during four judgment conditions on two emotional valences

\begin{tabular}{|c|c|c|c|c|c|c|c|c|}
\hline Conditions & Area & $\begin{array}{l}\text { Cluster } \\
\text { extent }\end{array}$ & $\begin{array}{l}\text { Brodmann's } \\
\text { area }\end{array}$ & Side & $\begin{array}{l}Z \\
\text { value }\end{array}$ & $x$ & $y$ & $z$ \\
\hline \multirow{5}{*}{$\begin{array}{l}\text { Other perspective self-judgment/ } \\
\text { positive (OS-P) }\end{array}$} & Cerebellum & 1217 & & Right & 6.22 & -26 & -80 & -34 \\
\hline & Precuneus & 2182 & 31 & Left & 6.17 & -6 & -56 & 34 \\
\hline & Angular gyrus & 1315 & 39 & Left & 5.51 & -44 & -60 & 30 \\
\hline & Superior medial frontal gyrus & 2997 & 10 & Left & 5.41 & -10 & 52 & 46 \\
\hline & Medial frontal gyrus & 559 & 8 & Left & 4.54 & -34 & 12 & 40 \\
\hline \multirow{5}{*}{$\begin{array}{l}\text { Other perspective self-judgment/ } \\
\text { negative (OS-N) }\end{array}$} & Posterior cingulum & 2142 & 23 & Left & 6.20 & -10 & -50 & 30 \\
\hline & Angular gyrus & 868 & 39 & Left & 5.58 & -40 & -58 & 30 \\
\hline & Superior medial frontal gyrus & 1200 & 8 & Left & 5.39 & -10 & 40 & 50 \\
\hline & Cerebellum & 456 & & Right & 5.32 & 26 & -80 & -34 \\
\hline & Medial frontal gyrus & 389 & 8 & Left & 4.17 & -34 & 12 & 42 \\
\hline \multirow{5}{*}{$\begin{array}{l}\text { Other perspective other judgment/ } \\
\text { positive (OO-P) }\end{array}$} & Cerebellum & 848 & & Right & 5.86 & 24 & -80 & -32 \\
\hline & Precuneus & 1841 & 31 & Left & 5.59 & -6 & -54 & 32 \\
\hline & Angular gyrus & 1128 & 39 & Left & 5.43 & -48 & -60 & 26 \\
\hline & Superior medial frontal gyrus & 2338 & 8 & Left & 5.28 & -8 & 52 & 42 \\
\hline & Precentral gyrus & 919 & 8 & Left & 4.75 & -40 & 12 & 46 \\
\hline \multirow{2}{*}{$\begin{array}{l}\text { Other perspective other judgment/ } \\
\text { negative (OO-N) }\end{array}$} & Angular gyrus & 1083 & 39 & Left & 6.48 & -42 & -58 & 28 \\
\hline & Precuneus & 2654 & 31 & Left & 6.21 & -6 & -58 & 26 \\
\hline \multirow{4}{*}{$\begin{array}{l}\text { Self-perspective self-judgment/ } \\
\text { positive (SS-P) }\end{array}$} & Calcarine & 1657 & & Right & 5.23 & 8 & -84 & 4 \\
\hline & Precuneus & 1221 & 31 & Left & 4.90 & -6 & -54 & 30 \\
\hline & Superior frontal gyrus & 1644 & 9 & Left & 4.66 & -8 & 56 & 28 \\
\hline & Caudate & 161 & & Left & 4.07 & -10 & 10 & 16 \\
\hline \multirow{3}{*}{$\begin{array}{l}\text { Self-perspective self-judgment/ } \\
\text { negative (SS-N) }\end{array}$} & Angular gyrus & 1083 & 39 & Left & 6.48 & -42 & -58 & 28 \\
\hline & Precuneus & 2654 & 31 & Left & 6.21 & -6 & -58 & 26 \\
\hline & Superior medial frontal gyrus & 912 & 10 & Left & 4.45 & -10 & 58 & 16 \\
\hline \multirow{4}{*}{$\begin{array}{l}\text { Self-perspective other judgment/ } \\
\text { positive (SO-P) }\end{array}$} & Precuneus & 2413 & 31 & Left & 6.97 & -6 & -58 & 32 \\
\hline & Angular gyrus & 1351 & 39 & Left & 5.77 & -44 & -60 & 26 \\
\hline & Superior medial frontal gyrus & 4667 & 10 & Left & 5.64 & -8 & 52 & 44 \\
\hline & Medial temporal pole & 1193 & 21 & Left & 5.10 & -56 & -8 & -24 \\
\hline \multirow{4}{*}{$\begin{array}{l}\text { Self-perspective other judgment/ } \\
\text { negative (SO-N) }\end{array}$} & Precuneus & 5589 & 31 & Left & 6.08 & -6 & -56 & 28 \\
\hline & Medial temporal pole & 373 & 20 & Left & 5.46 & -58 & -10 & -20 \\
\hline & Rectus gyrus & 1773 & 11 & Left & 5.43 & -4 & 44 & -14 \\
\hline & Angular gyrus & 483 & 39 & Left & 4.60 & -46 & -58 & 26 \\
\hline
\end{tabular}

region to assess possible functional connectivity related to the intervention effects under the OS condition. Seed regions were defined as radius spheres $(6 \mathrm{~mm})$ at specific coordinates based on the results of fMRI group analysis regarding the effect of BA. We created subtraction images of OS-P contrast (postintervention minus pre-intervention) which were extracted using gPPI of each subject. To determine whether there were any intervention effects on functional connectivity, these subtraction images were compared between intervention and control groups by using a two-sample $t$ test. We set the statistical threshold in the whole-brain analysis at $p<0.001$ (uncorrected) at the single voxel level, and $p<0.05$ (FWE-small volume corrected) at the cluster level. However, there were no significant differences in connectivity for coupled regions between pre- and post-intervention.

\section{Discussion}

In this study, we found increased activation in the $\mathrm{dmPFC}$ in OS-P after BA. Furthermore, there was a positive correlation between this activation increase and improvement of depressive symptoms. Additionally, and only in the OS-P, reaction times for self-referential judgments increased in the intervention group after BA, and there was a positive correlation between improvement of depressive symptoms and this increase. The present study is the first to reveal an effect of BA on other perspective self-reference, at both behaviour and brain-function levels. This pattern suggests that BA enhances adaptive self-monitoring.

Previous studies have suggested that while selfperspective self-reference reflects subjective mental images which arise in a moment (Markus, 1977), other perspective self-evaluation reflects objective 


$$
\text { Intervention group }(n=27)>\text { Control group }(n=29)
$$

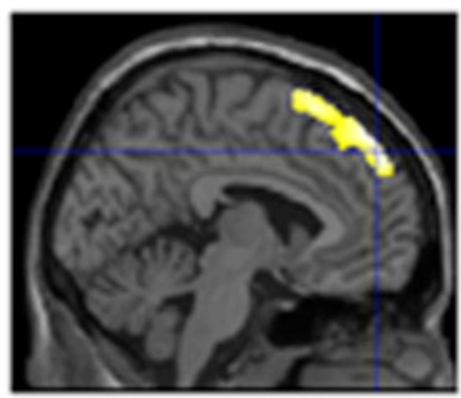

$$
x=-2
$$

\section{$t$ value}

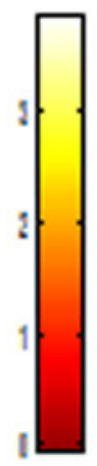

Fig. 1. Increase in left dorsal (superior) medial prefrontal cortex (mPFC) activation during the other perspective self-judgment (OS-P) condition for positive words. The intervention group showed significantly increased activation of the left dorsal (superior) $\operatorname{mPFC}(x=-2, y=48, z=50, t=3.24$, cluster size $=70$, cluster level family-wise error-corrected $p=0.016$, with small volume correction) compared with the control group.

mental images which come out through the autobiographical memory retrieval process (Ochsner et al. 2005). The self-perspective is assigned primarily during self-referential processing; however, when one evaluates the self from another's perspective, selfperspective self-referential processing is suppressed, and a monitoring function which views the self objectively is promoted (Yokoi et al. 2007).

According to Sheppard \& Teasdale (2000), healthy individuals show longer reaction times during difficult self-referential judgments, whereas people with depression do not differ in judgment reaction times as a function of difficulty, presumably due to faulty monitoring. Therefore, increased reaction times for other perspective self-referential processing in the intervention group might be related to BA-driven improvement in monitoring function. In BA, planning and executing goal-directed action is a primary focus, such that the treatment might lead to improvement in self-awareness of habitual behaviours or situational triggers, aspects of an objective monitoring function (Lewinsohn \& Grosscup, 1980; Jacobson et al. 1996).

Recent fMRI studies have suggested that the ventromedial PFC is involved in self-perspective selfreferential processing (Yoshimura et al. 2010, 2014), and that the dmPFC is involved in other perspective self-referential processing (Ruby \& Decety, 2001; Ochsner et al. 2005). In particular, our previous research (Yoshimura et al. 2014) reported that depressed individuals showed both decreased mPFC activation associated with reduced negative selfreference and increased $\mathrm{MPFC}$ activation associated with increased positive self-reference after CBT. We partially replicated these previous findings using BA as an intervention in the present study. In accordance with our prediction, we showed intervention effects on brain activity and behaviour indicators during positive self-reference (OS-P), but contrary to our prediction, not during negative self-reference (OS-N). This pattern might be due to the specific characteristics of the BA intervention as applied in the present study. During BA intervention, individuals might have positive experiences with a corresponding high frequency of environmental rewards and enhancement of monitoring, with improvements in monitoring function likely to be quite specific to such experiences. Therefore, intervention effects might be confined to the OS-P. In the present study, the more depressive symptom improvement a person showed, the longer the OS-P reaction times post-treatment. However, judgment ratio was not associated with depressive symptom improvement. Given these results, we speculated that change in self-concept might occur slowly, through self-evaluation using monitoring function. In contrast, utilization of monitoring function may be established immediately after BA, which might manifest as the increased reaction times we observed. However, as self-concept itself may not have yet changed, judgment ratios remained constant immediately post-treatment.

Regions of the mPFC might be related to aspects of cognitive control and scale with reaction time on task (Grinband et al. 2008). To exclude this possibility, we sought to confirm the relationship between interindividual variability and increased reaction times, and that inter-individual variability increased $\mathrm{mPFC}$ activation associated with OS-P. However, there were no significant positive or negative correlations. Therefore, we speculate that increased activation in the $\mathrm{mPFC}$ was not related to cognitive control associated with reaction times, and was associated with changes in other perspective self-referential processing. 

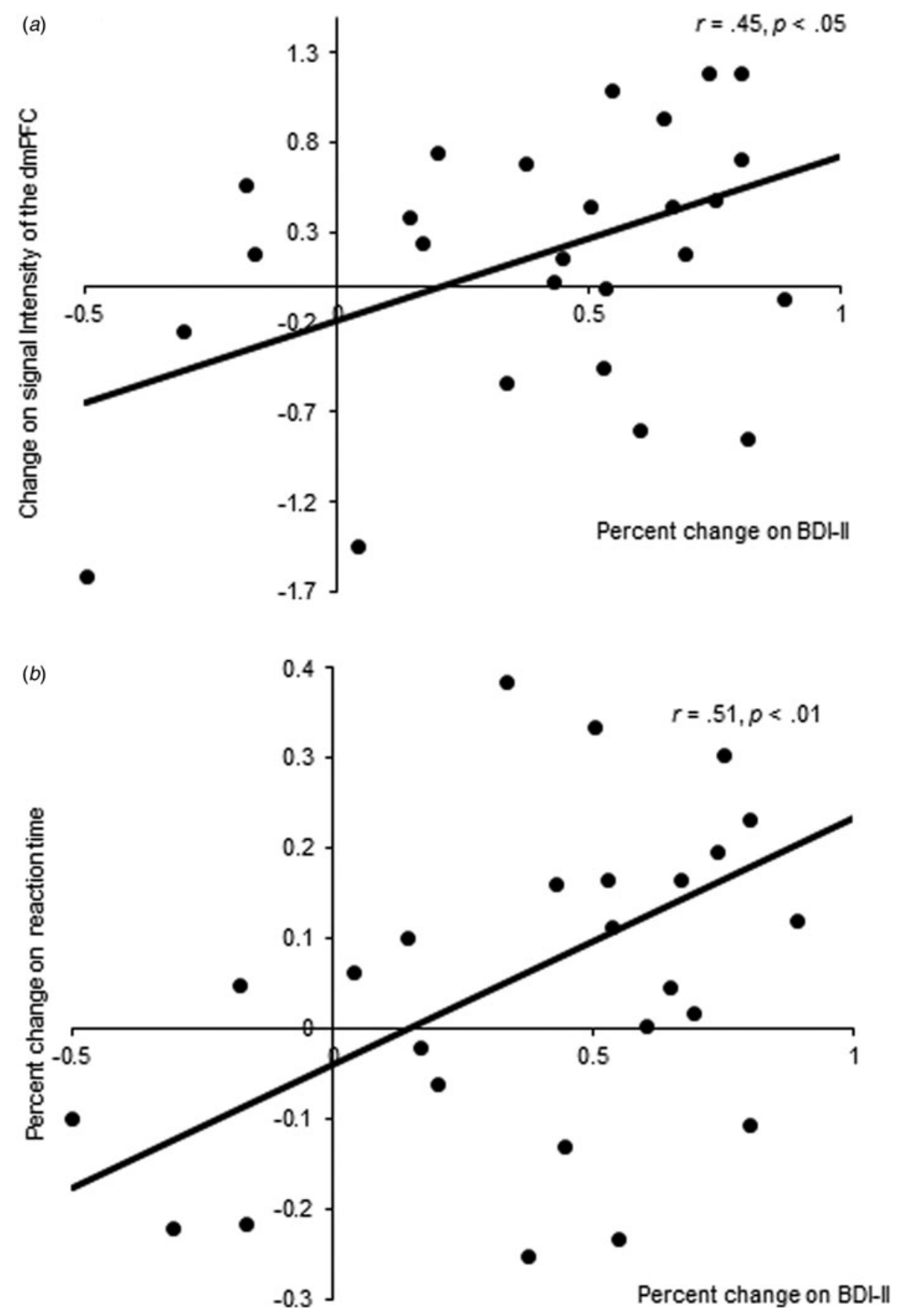

Fig. 2. Relationships between variables which show significant intervention effects. Scatter plots and associated correlation coefficients illustrate the relationship between increased dorsal medial prefrontal cortex (dmPFC) activation and percentage change on Beck Depression Inventory-II (BDI-II) scores during other perspective self-judgment condition for positive words (OS-P) in the intervention group (a). Scatter plots and associated correlation coefficients illustrate the relationship between percentage change on reaction times and percentage change on BDI-II scores during other perspective self-judgment condition for positive words (OS-P) in the intervention group $(b)$.

\section{Limitations}

There are several limitations of this study. First, we did not assess the monitoring function with any self-report questionnaires or observer-rated scales. Although the increased dmPFC activation and judgment latencies suggest improved monitoring function due to BA, quantitative evaluation of monitoring function would be more desirable.

Second, we conducted the present study without using healthy comparison participants. Although we cannot deny the specific effect of intervention, future 
research should take into consideration these issues. Despite several limitations, to our knowledge, this is the first intervention study to reveal neurobehavioural evidence for the effect of BA on subthreshold depression in self-referential processing.

Third, as shown in the online Supplementary material, there was a significant main effect of group on $\triangle$ brain activation in the $\mathrm{mPFC}$. There was significantly increased activation for each contrast in the mPFC of the intervention group than in the control group. We conducted a two-sample $t$ test (intervention group $v$. control group) on $\Delta$ brain activation in each judgment condition and emotional valence. Moreover, to correct for multiplicity of statistical tests, we report $p<0.00625$ $(0.05 / 8)$ for two-sample $t$ tests in each condition. Results indicated that the intervention group showed a significant increase in $\mathrm{mPFC}$ activation than the control group only in OS-P ( $p=0.00083)$. Additionally, we conducted a four-way mixed ANOVA on judgment ratio and reaction times, which indicated a significant interaction in judgment ratio between perspective and valence. Negative valence decreased significantly when using other perspective compared with selfperspective. We also found a significant main effect of reaction time on perspective, as well as a significant interaction between group, time and valence. Reaction time with referential processing using other perspective was longer than referential processing using selfperspective. Furthermore, reaction times increased in the intervention group compared with the control group after BA only in OS-P.

Furthermore, participants in the study were $67 \%$ male and $33 \%$ female, which was significantly different. According to previous studies, there are no gender differences in the prevalence estimates of subthreshold depression (Kessler \& Walters, 1998; Yang et al. 2010). In 2014, 2514 freshman were admitted to Hiroshima University, consisting of 1596 males (64\%) and 918 females $(36 \%)$. Of these, we randomly selected freshmen with a BDI-II score greater than or equal to 10 , such that the participants were $62.5 \%$ male and $37.5 \%$ female. Therefore, the gender ratio of the participants of the study reflected the gender ratio of the freshman population in the university in that year.

Finally, we noted the concern about the use of percentage change scores. There are some criticisms about using percentage change scores in parametric tests, because the score often has a non-normal, or a biased distribution. However, using percentage change scores also has the following merits. It can adjust for differences in the range of different variables (Tan \& Michel, 2011). Providing information related to the content and precision of scores helps the meaningful interpretation of raw scores (Tong \& Kolen, 2010). Analysis of data from clinical trials often uses percentage change scores and raw change scores to adjust treatment responses to the baseline (Kaiser, 1989). For these reasons, we used the percentage change score. The percentage change of BDI-II score and percentage change of reaction time scores in the intervention group and percentage change of reaction time scores in the control group were normally distributed. However, the percentage change score of BDI-II score in the control group was not normally distributed. Therefore, we cannot rule out the possibility that the percentage change score does not typically follow a normal distribution.

\section{Conclusions}

In summary, our results suggest that BA led to increased activation in the $\mathrm{dmPFC}$ during other perspective self-referential processing of positive trait words in people with subthreshold depression, which might contribute to improvement in depressive symptoms and objective monitoring function.

\section{Supplementary material}

The supplementary material for this article can be found at https://doi.org/10.1017/S0033291716002956

\section{Acknowledgements}

This work was supported (in part) by JSPS KAKENHI grant numbers JP16H06395, 16H06399, 23118004 and 26461748. Also, this research was partially supported by the Strategic Research Program for Brain Sciences from Japan Agency for Medical Research and Development, AMED (grant numbers: 15dm0107010h0005, 15dm0207012h0002 and 16dm0107093h0001).

\section{Declaration of Interest}

None.

\section{References}

Anderson NH (1968). Likableness rating of 555 personality-trait words. Journal of Personality and Social Psychology 9, 272-279.

Bertha EA, Balazs J (2013). Subthreshold depression in adolescence: a systematic review. European Child and Adolescent Psychiatry 22, 589-603.

Bochner S, Van-zyl T (1985). Desirability ratings of 110 personality-trait words. Journal of Social Psychology 125, 459-465.

Calni T, Sivers H, Thomason ME, Whitfield-Gabrieli S, Gabrieli JDE, Gotlib IH (2004). Brain activation to emotional words in depressed vs healthy subjects. Neuroreport 15, 2585-2588. 
Cuijpers P, Smit F, van Straten A (2007). Psychological treatments of subthreshold depression: a meta-analytic review. Acta Psychiatrica Scandinavica 115, 434-441.

Davis MH, Conklin L, Smith A, Luce C (1996). Effect of perspective taking on the cognitive representation of persons: a merging of self and other. Journal of Personality and Social Psychology 70, 713-726.

Dichter GS, Felder JN, Petty C, Bizzell J, Ernst M, Smoski MJ (2009). The effects of psychotherapy on neural responses to rewards in major depression. Biological Psychiatry 66, 886-897.

Dichter GS, Felder JN, Smoski MJ (2010). The effects of brief behavioral activation therapy for depression on cognitive control in affective contexts: an fMRI investigation. Journal of Affective Disorders 126, 236-244.

Galinsky AD, Ku G (2004). The effects of perspectivetaking on prejudice: the moderating role of self-evaluation. Personality and Social Psychology Bulletin 30, 594-604.

Grinband J, Wager TD, Lindquist M, Ferrera VP, Hirsch J (2008). Detection of time-varying signals in event-related fMRI designs. Neurolmage 43, 509-520.

Hammad TA, Laughren T, Racoosin J (2006). Suicidality in pediatric patients treated with antidepressant drugs. Archives of General Psychiatry 63, 332-339.

Hashimoto RI, Itahashi T, Ohta H, Yamada T, Kanai C, Nakamura M, Watanabe H, Kato N (2016). Altered effects of perspective-taking on functional connectivity during selfand other-referential processing in adults with autism spectrum disorder. Social Neuroscience. Published online 30 August 2016. doi:10.1080/17470919.2016.1224202.

Hollon SD, Kendall PC (1980). Cognitive self-statements in depression: development of an automatic thoughts questionnaire. Cognitive Therapy and Research 4, 383-395.

Jacobson NS, Dobson KS, Truax PA, Addis ME, Koerner K, Gollan JK, Prince SE (1996). A component analysis of cognitive-behavioral treatment for depression. Journal of Consulting and Clinical Psychology 64, 295-304.

Kaiser L (1989). Adjusting for baseline: change or percentage change? Statistics in Medicine 8, 1183-1190.

Kawakami N, Takeshima T, Ono Y, Uda H, Hata Y, Nakane Y, Nakane H, Iwata N, Furukawa T, Kikkawa T (2005). Twelve-month prevalence, severity, and treatment of common mental disorders in communities in Japan: preliminary finding from the World Mental Health Japan Survey 2002-2003. Psychiatry and Clinical Neuroscience 59, 441-452.

Kessler RC, Walters EE (1998). Epidemiology of DSM-III-R major depression and minor depression among adolescents and young adults in the National Comorbidity Survey. Depression and Anxiety 14, 3-14.

Kim HY (2013). Statistical notes for clinical researchers: assessing normal distribution (2) using skewness and kurtosis. Restorative Dentistry and Endodontics 38, 52-54.

Kojima M, Furukawa T (2003). Japanese Version of the Beck Depression Inventory, 2nd edn. Hyoron-sha Co.: Tokyo.

Kuiper NA, Derry PA (1982). Depressed and nondepressed content self-reference in mild depressives. Journal of Personality 50, 67-80.
Lewinsohn PM, Grosscup JM (1980). Changing reinforcing events: an approach to the treatment of depression. Psychotherapy: Theory, Research and Practice 17, 322-334.

Markus H (1977). Self-schemata and processing information about the self. Journal of Personality and Social Psychology 35, 63-78.

Mclaren DG, Ries ML, Xu G, Johnson SC (2012). A generalized form of context-dependent psychophysiological interactions (gPP): a comparison to standard approaches. NeuroImage 61, 1277-1286.

Mori A, Okamoto Y, Okada G, Takagaki K, Jinnin R, Takamura M, Kobayakawa M, Yamawaki S (2016). Behavioral activation can normalize neural hypoactivation in subthreshold depression during a monetary incentive delay task. Journal of Affective Disorders 189, 254-262.

Ochsner KN, Ray RD, Cooper JC, Robertson ER, Chopra S, Gabrieli JDE, Gross JJ (2005). The neural correlates of direct and reflected self-knowledge. NeuroImage 28, 797-814.

Rogers TB, Kuiper NA, Kirker WS (1977). Self-reference and the encoding of personal information. Journal of Personality and Social Psychology 35, 677-688.

Ruby P, Decety J (2001). Effect of subjective perspective taking during simulation of action: a PET investigation of agency. Nature Neuroscience 4, 546-550.

Russell M (2000). Summarizing change in test scores part I: shortcomings of three common methods. Practical Assessment, Research and Evaluation 7 (http://pareonline.net/ getvn.asp?v=7\&n=5). Accessed August 2016.

Sheppard LC, Teasdale JD (2000). Dysfunctional thinking in major depressive disorder: a deficit in metacognitive monitoring? Journal of Abnormal Psychology 109, 768-776.

Sugimoto N (2008). Basic Processing for Data. Introduction of Statistics for Learners of Medical Science, Pharmaceutical Sciences, Biological Science - the Basis and Practice of Data Analysis, p. 56. Pleiades Publishing: Osaka.

Surguladze SA, Young AW, Senior C, Travis MJ, Phillips ML (2004). Recognition accuracy and response bias to happy and sad facial expressions in patients with major depression. Neuropsychology 18, 212-218.

Symons CS, Johnson BT, Symons CS, Johnson BT (1997) The self-reference effect in memory: a meta- analysis the self-reference effect in memory: a meta-analysis. Psychological Bulletin 121, 371-394.

Takagaki K, Okamoto $Y$, Jinnin R, Mori A, Nishiyama $Y$, Yamamura T, Yokoyama S, Shiota S, Okamoto $Y$, Miyake Y, Ogata A, Kunisato Y, Shimoda H, Kawakami N, Furukawa A, Yamawaki S (2016). Behavioral activation for late adolescents with subthreshold depression: a randomized controlled trial. European Child and Adolescent Psychiatry 25, 1171-1182.

Tan X, Michel R (2011). Why do standardized testing programs report scaled scores? Why not just report the raw or percent-correct scores? $R$ \& D Connections 16, 1-6.

Tong Y, Kolen MJ (2010). Scaling: an ITEMS module. Educational Measurement: Issues and Practice 29, 39-48.

Weightman MJ, Air TM, Baune BT (2014). A review of the role of social cognition in major depressive disorder. Frontiers in Psychiatry 5, 179. 
Yang J, Yao S, Zhu X, Zhang C, Ling Y, Abela JRZ, Esseling PG, Mcwhinnie C (2010). The impact of stress on depressive symptoms is moderated by social support in Chinese adolescents with subthreshold depression: a multi-wave longitudinal study. Journal of Affective Disorders 127, 113-121.

Yokoi T, Takemura N, Ogawa K, Inui T (2007). Neural substrates for inference of other's mind based on perspective taking and information suppression. Institute of Electronics, Information and Communication Engineers 41, 45-50.
Yoshimura S, Okamoto Y, Onoda K, Matsunaga M, Okada G, Kunisato Y, Yamawaki S (2014). Cognitive behavioral therapy for depression changes medial prefrontal and ventral anterior cingulate cortex activity associated with self-referential processing. Social Cognitive and Affective Neuroscience 9, 487-493.

Yoshimura S, Okamoto Y, Onoda K, Matsunaga M, Ueda K (2010). Rostral anterior cingulate cortex activity mediates the relationship between the depressive symptoms and the medial prefrontal cortex activity. Journal of Affective Disorders 122, 76-85. 